\title{
Opacity of an Automaton. Application to the Inhomogeneous Ising Chain
}

\section{Mendes France}

Département de Mathématiques, Université Bordeaux I, F-33405 Talence Cedex, France

Received November 8, 1990; in revised form January 31, 1991

Abstract. An automaton maps infinite sequences onto infinite sequences. We define the opacity as the distance between output sequences and input sequences. A transparent automaton hardly disturbs the input sequence. An opaque automaton erases some of the information contained in the input sequence. We apply these ideas to the study of the inhomogeneous Ising chain governed by the Hamiltonian

$$
\mathscr{H}_{\varepsilon}(\sigma)=-J \sum_{q=-M}^{N-1} \varepsilon_{q} \sigma_{q} \sigma_{q+1}-H \sum_{q=-M}^{N} \sigma_{q}
$$

\section{Part One. Automata}

\section{I.1. Definition of an Automaton}

Problems in one-dimension physics often depend on the solution of a recurrence relation of the type

$$
\delta_{n+1}=f\left(\delta_{n}\right),
$$

where $f$ is a given map and where $\delta_{0}$ is known. It may happen that the function $f$ itself varies from step to step. For example, suppose we are given two functions $f_{+}$ and $f_{-}$and a sequence of signs $\left(\varepsilon_{n}\right), \varepsilon_{n} \in\{-,+\}$. Then the sequence $\left(\delta_{n}\right)$ defined by

$$
\delta_{n+1}=f_{\varepsilon_{n}}\left(\delta_{n}\right)
$$

depends on the sequence $\left(\varepsilon_{n}\right)$. This situation arises in the study of the inhomogeneous Ising Chain where the coupling constant varies from one site to the other (binary alloy, chain with impurities, spin glass, ...).

The object of the paper is to give a general discussion of the $\operatorname{map}\left(\varepsilon_{n}\right) \mapsto\left(\delta_{n}\right)$ and this can be achieved in terms of automata theory. We then apply our results to the Ising Chain. 
In the future, we hope to be able to adapt our techniques to the study of the discrete Schrödinger equation

$$
\delta_{n+2}-2 \delta_{n+1}+\delta_{n}=\left(\varepsilon_{n}-\lambda\right) \delta_{n+1} .
$$

The sequence $\left(\varepsilon_{n}\right)$ represents then a given potential and $\left(\delta_{n}\right)$ the time independent wave function.

We now define an automaton. The automata we wish to discuss are of a special kind. They map the set $\{-1,+1\}^{\mathbb{N}}$ of infinite $( \pm)$ sequences into the set $\mathscr{R}^{\mathbb{N}}$ of real sequences as follows.

We are given a finite set $S$ of states $\mathrm{A}, \mathrm{B}, \mathrm{C}, \mathrm{D}, \ldots$ and among them an initial state, say $A$, two maps $f_{+}: S \rightarrow S$ and $f_{-}: S \rightarrow S$ represented by arrows, and an output function $\varphi: S \rightarrow \mathscr{R}$. The automation $\mathscr{A}$ is the quadruple $\left(S, f_{+}, f_{-}, \varphi\right)$. It will be convenient to simplify notations and to write $+A,+B, \ldots$ (respectively $-A$, $-B, \ldots$ ) instead of $f_{+}(A), f_{+}(B), \ldots$ (respectively $\left.f_{-}(A), f_{-}(B), \ldots\right)$.

Given the input, i.e. an infinite sequence $\varepsilon=\left(\varepsilon_{0}, \varepsilon_{1}, \ldots\right)$ of $(+)$ and $(-)$ symbols, it acts on $\mathscr{A}$ producing the sequence $\varepsilon_{0} A, \varepsilon_{1} \varepsilon_{0} A, \varepsilon_{2} \varepsilon_{1} \varepsilon_{0} A, \ldots$ We read off

$$
\delta_{0}=\varphi\left(\varepsilon_{0} A\right), \delta_{1}=\varphi\left(\varepsilon_{1} \varepsilon_{0} A\right), \delta_{2}=\varphi\left(\varepsilon_{2} \varepsilon_{1} \varepsilon_{0} A\right), \ldots
$$

The infinite real sequence $\delta=\left(\delta_{0}, \delta_{1}, \ldots\right)$, denoted $\mathscr{A} \varepsilon$, is called the transduced sequence of $\varepsilon$.

The reader may wonder why it is essential to introduce the output function $\varphi$. The reason will appear in the next paragraph where we define equivalent automata, a notion which will prove useful in defining the opacity.

Automata can be advantageously represented as oriented graphs where from each vertex (state) two arrows $(+$ and - ) leave. We illustrate the definition by three examples.

Example 1. (The one state automaton)

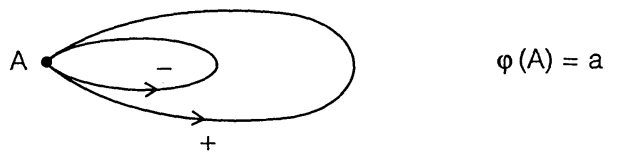

All $( \pm)$ sequences are mapped onto the constant sequence $(a, a, a, \ldots)$.

Example 2. (An identity automaton)

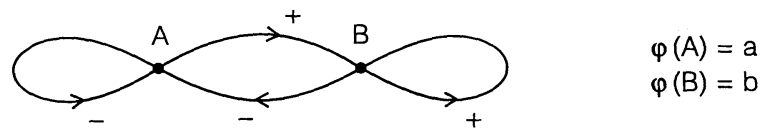

This automaton acts as the "identity." For example the sequence $-++-+-\ldots$ is mapped onto $a b b a b a \ldots$ The symbol $(-)$ is coded onto a and (+) onto $b$. Had the output function been $\varphi(A)=-1, \varphi(B)=+1$, then $\mathscr{A} \varepsilon=\varepsilon$.

Example 3. (Thue-Morse automaton)

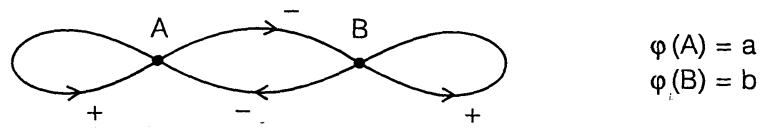


Let $\varepsilon=\left(\varepsilon_{0}, \varepsilon_{1}, \ldots\right)$ be an input sequence. Define

$$
\pi_{n}=\varepsilon_{0} \varepsilon_{1} \ldots \varepsilon_{n} .
$$

Then the output sequence $\delta=\left(\delta_{0}, \delta_{1}, \ldots\right)$ is

$$
\delta_{n}=\left\{\begin{array}{lll}
a & \text { if } & \pi_{n}=+1 \\
b & \text { if } & \pi_{n}=-1 .
\end{array}\right.
$$

In other terms

$$
\delta_{n}=\frac{1}{2}(a+b)+\frac{1}{2}(a-b) \pi_{n} .
$$

In particular, if $a=+1$ and $b=-1$, then $\delta_{n}=\pi_{n}$.

\section{I.2. Further Definitions}

Two automata $\mathscr{A}$ and $\mathscr{A}^{\prime}$ are said to be equivalent $\left(\mathscr{A} \sim \mathscr{A}^{\prime}\right)$ if they differ at most by their output function.

A null-automaton sends all input sequences onto the null sequence $(0,0,0, \ldots)$. Every automaton is equivalent to some null-automaton. The corresponding output function is the null-function $\varphi(A)=\varphi(B)=\varphi(C)=\ldots=0$.

In an automaton, a state falls in one of the four categories:

(i) it only has incident $(+)$ arrows,

(ii) it only has incident (-) arrows,

(iii) it has (+) and (-) incident arrows,

(iv) it has no incident arrows.

An automaton is called balanced if the output function $\varphi$ maps all states of the first kind onto +1 , all states of the second kind onto -1 and all states of the third and fourth kind onto 0. Every automaton is mapped onto some balanced automaton.

In our previous Example 1, the equivalent balanced automata is defined by $\varphi(A)=0$; in Example 2, $\varphi(A)=-1, \varphi(B)=+1$; in Example 3, $\varphi(A)=\varphi(B)=0$.

An automaton is said to be homogeneous if there are exactly two incident arrows to each state. This is the case in Examples 1, 2, and 3. Example 4 below shows an automaton which is not homogeneous.

An automaton is never empty: it always possesses at least an initial state and two arrows. A subautomaton of an automaton $\mathscr{A}$ is obtained by deleting a family of its states together with the incident and departing arrows corresponding to these states. An automaton may well have no subautomaton but itself. Such is the case in Examples 1, 2, and 3.

Example 4.

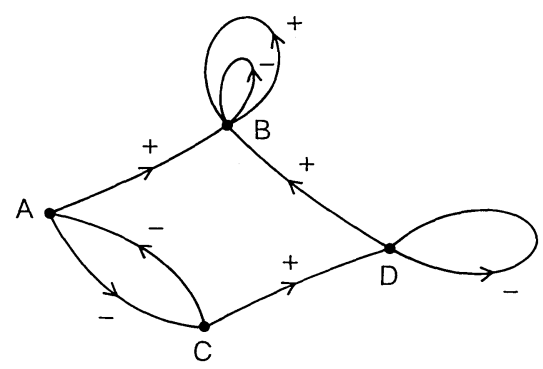


Suppressing $A$ does not leave us with an automaton. But suppressing $A$ and $C$ leaves the two state automaton:

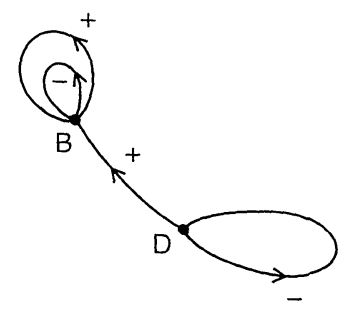

Suppressing $A, C$, and $D$ leaves us with the one state automaton

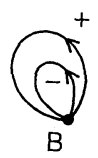

So the initial automaton possesses at least two proper subautomata. One should note that the initial state of the subautomata can be chosen arbitrarily.

\section{I.3. Opacity of an Automaton}

We propose to measure the distortion that an automaton produces on inputs. If the output sequences contain all the features of the input sequences we would like to think of transparency. If not the automaton is opaque (or partially opaque).

The norm of a real bounded sequence $\sigma=\left(\sigma_{0}, \sigma_{1}, \ldots\right)$ is

$$
\|\sigma\|=\limsup _{N \rightarrow \infty}\left(\frac{1}{N}\left(\sum_{n=0}^{N-1}\left|\sigma_{n}\right|^{2}\right)\right)^{1 / 2} .
$$

The distance between two real bounded sequences $\sigma$ and $\sigma^{\prime}$ is thus

$$
\left\|\sigma-\sigma^{\prime}\right\|=\limsup _{N \rightarrow \infty}\left(\frac{1}{N}\left(\sum_{n=0}^{N-1}\left|\sigma_{n}-\sigma_{n}^{\prime}\right|^{2}\right)\right)^{1 / 2} .
$$

The exponents 2 and $1 / 2$ that appear in the above definitions are not really essential. They could be replaced by $p$ and $1 / p(p \geqq 1)$. The choice $p=2$ has however a small advantage in that it is often easier to compute the norm in a more analytical fashion.

The distance between $\sigma$ and $\sigma^{\prime}$ may well vanish even though $\sigma$ and $\sigma^{\prime}$ are not identical. For example, $\sigma$ and $\sigma^{\prime}$ may differ from each other on finitely many elements only. Yet we shall consider such sequences as equal....

The opacity of an automaton $\mathscr{A}$ is defined as

$$
\omega(\mathscr{A})=\sup _{\varepsilon} \inf _{\mathscr{A}^{\prime} \sim \mathscr{A}}\left\|\mathscr{A}^{\prime} \varepsilon-\varepsilon\right\|,
$$

where the sup is taken over all ultimately periodic $( \pm)$ sequences $\varepsilon$.

Let us illustrate this formula. First of all, we observe that

$$
\left\|\mathscr{A}^{\prime} \varepsilon-\varepsilon\right\| \leqq\left\|\mathscr{A}^{\prime} \varepsilon\right\|+\|\varepsilon\|=\left\|\mathscr{A}^{\prime} \varepsilon\right\|+1
$$


so that

$$
\omega(\mathscr{A}) \leqq 1+\sup _{\varepsilon} \inf _{\mathscr{A}^{\prime}}\left\|\mathscr{A}^{\prime} \varepsilon\right\|
$$

The infimum is obviously attained for the null-automaton $\mathscr{A}^{\prime}$ equivalent to $\mathscr{A}$. Therefore

$$
0 \leqq \omega(\mathscr{A}) \leqq 1 \text {. }
$$

A transparent automaton corresponds to $\omega=0$ and for an opaque automaton $\omega=1$. We shall see later on that for any given rational $r \in[0,1]$ there exists an automaton $\mathscr{A}$ for which

$$
\omega(\mathscr{A})=\sqrt{r} .
$$

Conversely $\omega(\mathscr{A})$ is necessarily the square root of a rational number.

It is obvious from the definition that

$$
\mathscr{A} \sim \mathscr{A}^{\prime} \Rightarrow \omega(\mathscr{A})=\omega\left(\mathscr{A}^{\prime}\right) .
$$

The following obvious inequalities can be useful to calculate $\omega(\mathscr{A})$ :

$$
\begin{aligned}
& \omega(\mathscr{A}) \geqq \inf _{\mathscr{A}^{\prime} \sim \mathscr{A}}\left\|\mathscr{A}^{\prime} \varepsilon-\varepsilon\right\| \text { for all ultimately periodic sequences } \varepsilon, \\
& \omega(\mathscr{A}) \leqq \sup _{\varepsilon}\left\|\mathscr{A}^{\prime} \varepsilon-\varepsilon\right\| \text { for all } \mathscr{A}^{\prime} \sim \mathscr{A} .
\end{aligned}
$$

Example 1. (The one state automaton).

Choose $\varepsilon_{n}=(-1)^{n}$. Then

$$
\omega^{2}(\mathscr{A})=\sup _{\varepsilon} \inf _{a \in \mathscr{R}} \limsup _{N \rightarrow \infty} \frac{1}{N}\left[\sum_{\substack{n<N \\ \varepsilon_{n}=+1}}(a-1)^{2}+\sum_{\substack{n<N \\ \varepsilon_{n}=-1}}(a+1)^{2}\right] .
$$

$$
\omega^{2}(\mathscr{A}) \geqq \inf _{a \in \mathscr{R}} \frac{1}{2}\left[(a-1)^{2}+(a+1)^{2}\right] .
$$

The infimum is obtained for $a=0$ :

$$
\omega^{2}(\mathscr{A}) \geqq 1,
$$

hence

$$
\omega(\mathscr{A})=1
$$

The automaton is as opaque as it can be. In this case, the output gives no information on the input. In Example 3 we shall see an opaque automaton for which the situation is less "dramatic."

Example 2. (Identity automaton).

$$
\omega^{2}(\mathscr{A})=\sup _{\varepsilon} \inf _{a, b} \limsup _{N \rightarrow \infty} \frac{1}{N}\left[\sum_{\substack{n<N \\ \varepsilon_{n}=+1}}(b-1)^{2}+\sum_{\substack{n<N \\ \varepsilon_{n}=-1}}(a+1)^{2}\right] .
$$

The infimum is attained for $a=-1$ and $b=+1$. Hence $\omega(\mathscr{A})=0$. The identity automaton is transparent, as expected.

Example 3. (Thue-Morse automaton). Let $\varepsilon$ be $(-1)^{n}$. We know that the output seqence is

$$
\delta_{n}=\frac{1}{2}(a+b)+\frac{1}{2}(a-b) \pi_{n} .
$$


We now compute $\|\delta-\varepsilon\|$ :

$$
\|\delta-\varepsilon\|^{2}=\limsup _{N \rightarrow \infty} \frac{1}{N} \sum_{n=0}^{N-1}\left(c+c^{\prime} \pi_{n}-\varepsilon_{n}\right)^{2},
$$

where $c=\frac{1}{2}(a+b), c^{\prime}=\frac{1}{2}(a-b)$. The limit actually exists and

$$
\|\delta-\varepsilon\|^{2}=\lim _{N \rightarrow \infty} \frac{1}{N} \sum_{n=0}^{N-1}\left[c^{2}+c^{\prime 2}+1+2 c c^{\prime} \pi_{n}-2 c \varepsilon_{n}-2 c^{\prime} \varepsilon_{n} \pi_{n}\right] .
$$

Then the three following averages vanish:

$$
\begin{aligned}
& \lim _{N \rightarrow \infty} \frac{1}{N} \sum_{n=0}^{N-1} \pi_{n}=0, \\
& \lim _{N \rightarrow \infty} \frac{1}{N} \sum_{n=0}^{N-1} \varepsilon_{n}=0, \\
& \lim _{N \rightarrow \infty} \frac{1}{N} \sum_{n=0}^{N-1} \varepsilon_{n} \pi_{n}=0 .
\end{aligned}
$$

Therefore

$$
\|\delta-\varepsilon\|^{2}=c^{2}+c^{\prime 2}+1
$$

and

$$
\omega(\mathscr{A}) \geqq \inf _{c, c^{\prime}}\left(c^{2}+c^{\prime 2}+1\right)=1 .
$$

Hence

$$
\omega(\mathscr{A})=1 .
$$

The automaton is opaque even though, contrary to Example 1, it is possible to compute $\varepsilon$ knowing $\delta=\mathscr{A} \varepsilon$. Yet the two sequences $\varepsilon$ and $\mathscr{A} \varepsilon$ may behave very differently. It can be shown for example that $\varepsilon$ can be almost-periodic whereas $\mathscr{A} \varepsilon$ is pseudo-random in the sense of Bass [2].

In this Example 3 we have used the $L^{2}$ structure to calculate $\omega(\mathscr{A})$. In the following paragraph we shall describe another method of calculating $\omega(\mathscr{A})$ which is more general and completely algorithmical.

\section{I.4. Computing the Opacity}

Let $P$ be an oriented path on the automaton and let $\lambda(P)$ be its length (number of arrows). Every automaton has at least one closed path (cyclic path). A state is said to belong strongly to a path if the path visits the state at least twice, once through a $(+)$ arrow and once through a $(-)$ arrow. Let $v(P)$ be the number of states that strongly belong to $P$.

Theorem 1. The opacity of a homogeneous automaton $\mathscr{A}$ is given by the formula

$$
\omega(\mathscr{A})=\sup _{\operatorname{closed} P} \sqrt{\frac{2 v(P)}{\lambda(P)}} .
$$


Proof. Let $P$ be a closed path of length $\lambda=\lambda(P)$ and let $A_{1}, A_{2}, \ldots, A_{v}$ be the $v=v(P)$ states that strongly belong to $P ; v$ may well be zero. Let $\varepsilon=\left(\varepsilon_{0}, \varepsilon_{1}, \ldots\right)$ be an ultimately periodic sequence (period $\lambda$ ) which from some point on follows the path $P$ twice through $A_{1}, A_{2}, \ldots, A_{v}$ and back to $A_{1}$. Let $\varphi$ be the output function and put $\varphi\left(A_{i}\right)=a_{i}$. Then

$$
\|\mathscr{A} \varepsilon-\varepsilon\|^{2} \geqq \frac{1}{\lambda}\left[\sum_{\substack{\varepsilon_{i}=+1 \\ i \leqq \nu}}\left(a_{i}-1\right)^{2}+\sum_{\substack{\varepsilon_{i}=-1 \\ i \leqq \nu}}\left(a_{i}+1\right)^{2}\right] .
$$

The inequality sign comes from the fact that the path $P$ may contain states which do not strongly belong to $P$. Because $\mathscr{A}$ is homogeneous, the right-hand side reads

$$
\frac{1}{\lambda} \sum_{i \leqq v}\left[\left(a_{i}-1\right)^{2}+\left(a_{i}+1\right)^{2}\right] \text {. }
$$

The infimum over all equivalent automata is obtained for $a_{i}=0$. Therefore

and

$$
\omega^{2}(\mathscr{A}) \geqq \frac{2 v}{\lambda}
$$

$$
\omega^{2}(\mathscr{A}) \geqq \sup _{P} \frac{2 v(P)}{\lambda(P)} .
$$

We now show that $\omega^{2}(\mathscr{A})$ cannot be larger than the sup. Let $\mathscr{A}^{\prime}$ be the balanced automaton equivalent to $\mathscr{A}$. Let the states be $A_{1}, A_{2}, \ldots$. For a given ultimately periodic $\varepsilon=\left(\varepsilon_{0}, \varepsilon_{1}, \ldots\right)$ the sequence of visited states is $A_{i_{0}}, A_{i_{1}}, A_{i_{2}}, \ldots$ Then

$$
\left\|\mathscr{A}^{\prime} \varepsilon-\varepsilon\right\|^{2}=\limsup _{N \rightarrow \infty} \frac{1}{N} \sum_{n<N}\left|\varphi\left(A_{i_{n}}\right)-\varepsilon_{n}\right|^{2} .
$$

If $A_{i_{n}}$ belongs strongly to the infinite path $\hat{P}$ generated by $\varepsilon$ then $\phi\left(A_{i_{n}}\right)=0$. If not, $\varphi\left(A_{i_{n}}\right)=\varepsilon_{n}$ so that

$$
\left\|\mathscr{A}^{\prime} \varepsilon-\varepsilon\right\|^{2}=\limsup _{N \rightarrow \infty} \frac{1}{N} \sum_{n<N}^{0} 1
$$

where the symbol $\circ$ indicates that the summation is extended to those $n$ for which $A_{i_{n}}$ belongs strongly to the path. Then

$$
\left\|\mathscr{A}^{\prime} \varepsilon-\varepsilon\right\|^{2} \leqq \frac{2 v\left(P_{\varepsilon}\right)}{\lambda\left(P_{\varepsilon}\right)}
$$

for some closed path $P_{\varepsilon}$ which depends on $\varepsilon$.

Therefore

$$
\begin{aligned}
\omega^{2}(\mathscr{A}) & \leqq \sup _{\varepsilon}\left\|\mathscr{A}^{\prime} \varepsilon-\varepsilon\right\| \leqq \sup _{\varepsilon} \frac{2 v\left(P_{\varepsilon}\right)}{\lambda\left(P_{\varepsilon}\right)} \\
& \leqq \sup _{\text {closed } P} \frac{2 v(P)}{\lambda(P)} \quad \text { Q.E.D. }
\end{aligned}
$$

The proof of the theorem implies the following corollaries.

Corollary 1. If $\mathscr{A}^{\prime}$ is the balanced automaton equivalent to a homogeneous automaton $\mathscr{A}$, then

$$
\omega(\mathscr{A})=\sup _{\varepsilon}\left\|\mathscr{A}^{\prime} \varepsilon-\varepsilon\right\| .
$$


Corollary 2. The range of $\omega^{2}(\mathscr{A})$ for homogeneous $\mathscr{A}$ is the set of rationals on $[0,1]$.

Using the theorem, it is easy to see that the automata of Examples 1, 2, and 3 have indeed opacities respectively equal to 1,0 and 1 as we had already seen. The automaton of Example 4 has opacity 1.

\section{Part Two. The Inhomogeneous Ising Chain}

\section{II.1. The Inhomogeneous Ising Chain}

An Ising chain is formed by $M+N$ particles in a row. Particles have spin \pm 1 . We denote the spin at site $q$ by $\sigma_{q}(q=-M,-M+1, \ldots, N)$. Let $\varepsilon \in\{-1,+1\}^{\bar{M}+N}$ be given. The Hamiltonian or energy of a given configuration of spins

$$
\sigma=\left(\sigma_{-M}, \sigma_{-M+1}, \ldots, \sigma_{N-1}, \sigma_{N}\right)
$$

is

$$
\mathscr{H}(\sigma)=-J \sum_{q=-M}^{N-1} \varepsilon_{q} \sigma_{q} \sigma_{q+1}-H \sum_{q=-M}^{N} \sigma_{q},
$$

where $J>0$ and $H \in \mathscr{R}$ are fixed parameters; $J$ is known as the coupling constant and measures the binding energy between two neighboring particles $q, q+1$, and $H$ is the external field which we shall always suppose nonnegative without loss of generality. The problem is to find the equilibrium configurations, i.e. those $\sigma$ which minimize $\mathscr{H}(\sigma)$. This turns out to be quite intricate and we have decided to tackle only one particular part of the problem. We let $M$ go to $+\infty$ and determine the spin $\sigma_{N}$ of the endpoint in equilibrium. $\sigma_{N}$ depends on $\sigma_{N-1}, \sigma_{N-2}, \ldots$. In other terms, we are discussing an Ising chain in which the interactions are one-sided. It then can be shown $[1,3]$ that $\sigma_{n}$ is the sign of $\delta_{n}$ where $\delta_{n}$ is defined inductively through the formula

$$
\delta_{n+1}=\frac{2 H}{J}+\varepsilon_{n} \operatorname{sgn}\left(\delta_{n}\right) \min \left\{2,\left|\delta_{n}\right|\right\}, \quad n<N .
$$

It can also be shown that for large $n, \delta_{n}$ does not depend on the value of $\delta_{0}$, except maybe for some very special sequence $\varepsilon$ (see [4]).

So from now on, we study the recurrence formula

$$
\left\{\begin{array}{l}
\delta_{0}=\alpha+2, \quad \alpha \geqq 0 \\
\delta_{n+1}=\alpha+\varepsilon_{n} \operatorname{sgn}\left(\delta_{n}\right) \min \left\{2,\left|\delta_{n}\right|\right\},
\end{array}\right.
$$

where we have put $\alpha=2 H / J$. To every ( \pm ) sequence $\varepsilon=\left(\varepsilon_{n}\right)$ corresponds a sequence $\delta=\left(\delta_{n}\right)$. What is this correspondence? This is the question we apply ourselves to.

\section{II.2. The Ising Automata}

It is easily verified that for fixed $\alpha$, the sequence $\delta=\left(\delta_{n}\right)$ can only take finitely many values which all lie in the interval $[\alpha-2, \alpha+2]$. The map $\varepsilon \mapsto \delta$ is an automaton $\mathscr{A}_{\alpha}$ 
which we shall now describe. (In a previous article [4], we used the word "transducer" rather than "automaton.")

Before embarking on the general case, let us look at some examples.

Example 5. $\alpha=0$. Then

$$
\left\{\begin{array}{l}
\delta_{n+1}=\varepsilon_{n} \operatorname{sgn}\left(\delta_{n}\right) \min \left\{2,\left|\delta_{n}\right|\right\} \\
\delta_{0}=2
\end{array} .\right.
$$

By looking at the first values of $\delta_{n}$, one can easily convince oneself that

$$
\delta_{n}=2 \varepsilon_{0} \varepsilon_{1} \ldots \varepsilon_{n-1},
$$

and this is just the Thue-Morse automaton of Example 3 with $\varphi(A)=2$ and $\varphi(B)$ $=-2$.

Example 6. $\alpha=2$. The inequalities $\alpha-2 \leqq \delta_{n} \leqq \alpha+2$ show that $0 \leqq \delta_{n}$ so that the induction formula boils down to

$$
\delta_{n+1}=2+\varepsilon_{n} \min \left\{2, \delta_{n}\right\} .
$$

The graphical representation of $\mathscr{A}_{2}$ is thus as follows.

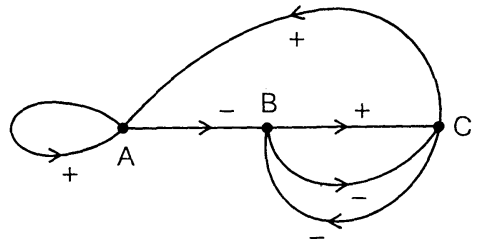

$$
\begin{aligned}
& \varphi(A)=4 \\
& \varphi(B)=0 \\
& \varphi(C)=2
\end{aligned}
$$

Example 7. $\alpha \geqq 4$. Then $\delta \geqq 2$. The induction formula reads

$$
\delta_{n+1}=\alpha+2 \varepsilon_{n},
$$

and we readily recognize the identity automaton

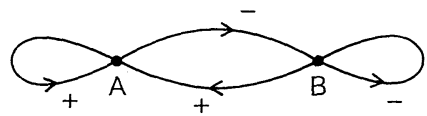

$$
\begin{aligned}
& \varphi(A)=\alpha+2 \\
& \varphi(B)=\alpha-2
\end{aligned}
$$

[There is a slight discrepancy with Example 2: arrows $(+)$ and $(-)$ are exchanged.]

Following Allouche, we now look at the general case which subdivides into two cases according to whether $4 / \alpha$ is or is not an integer. The cases $\alpha=0$ and $\alpha \geqq 4$ have already been treated.

Case where $4 / \alpha$ is not an integer.

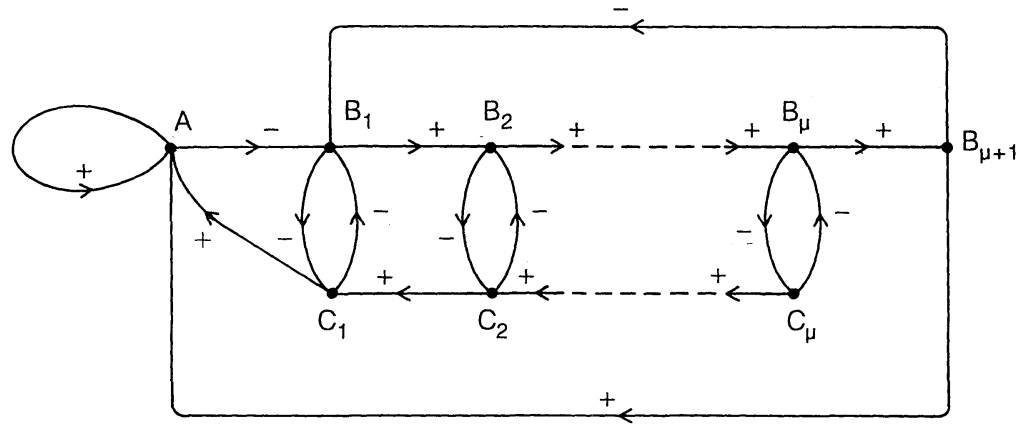




$$
\left\{\begin{array}{l}
\varphi(A)=\alpha+2 \\
\varphi\left(B_{i}\right)=i \alpha-2 \\
\varphi\left(C_{i}\right)=2-(i-1) \alpha, \quad \mu=\left[\frac{4}{\alpha}\right]
\end{array}\right.
$$

Case where $4 / \alpha$ is an integer.

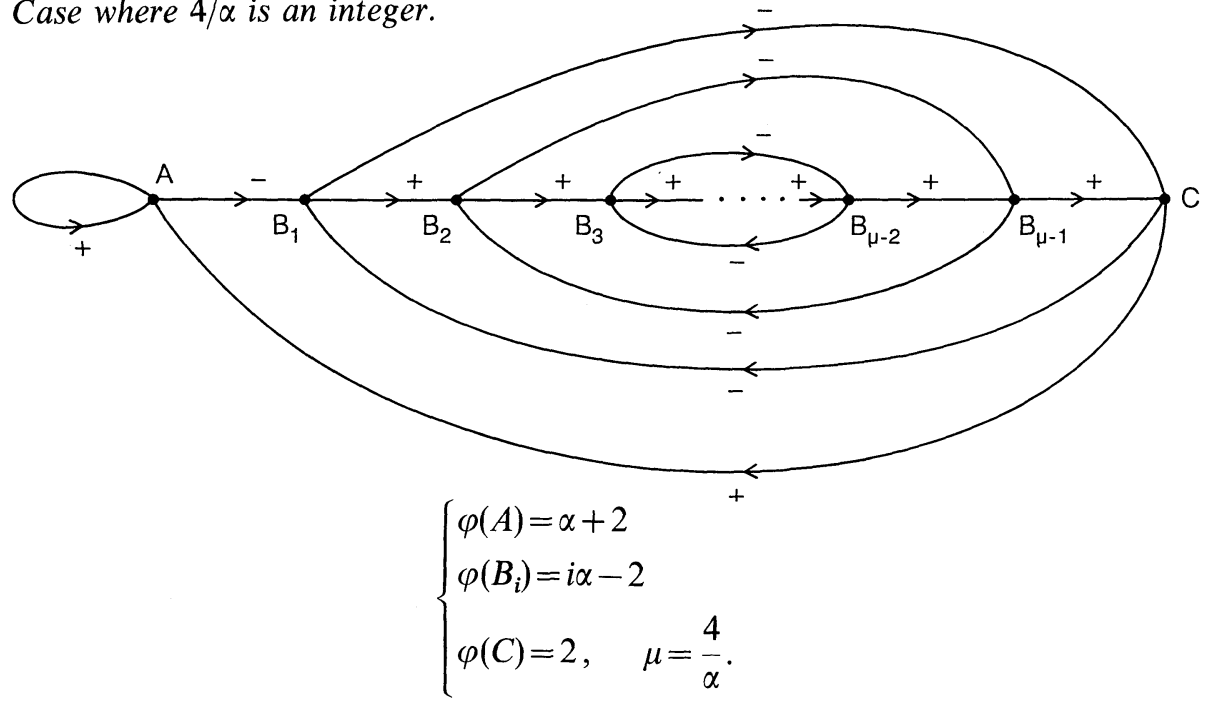

In this case, the Ising automaton $\mathscr{A}_{\alpha}$ is homogeneous.

Theorem 2. Let $S_{\alpha}$ be the set of states of the automaton $\mathscr{A}_{\alpha}$ and let $\left|S_{\alpha}\right|$ be the number of states. Then

$$
\frac{1}{\left|S_{\alpha}\right|} \sum_{M \in S_{\alpha}} \varphi(M)=\alpha .
$$

Proof. The proof is obvious for $\alpha=0$. If $\alpha \neq 0$ and if $4 / \alpha$ is not an integer then

$$
\left|S_{\alpha}\right|=2+2 \mu
$$

and the statement is easily verified.

If $4 / \alpha$ is an integer, then

$$
\left|S_{\alpha}\right|=\mu+1 \text {, }
$$

and again the proof is easily completed. Q.E.D.

Remark. When $\alpha$ decreases to 0 , the number of states tends to infinity. For $\alpha=0$ the number of states is 2 . The automaton $\mathscr{A}_{0}$ appears as singular.

The set $\{-1,+1\}^{\mathbf{N}}$ is canonically endowed with the Bernoulli measure

$$
d \varepsilon=\prod_{k \in \mathbb{N}} d \varepsilon_{k}=\prod_{k \in \mathbb{N}}\left(\frac{1}{2} D_{+}+\frac{1}{2} D_{-}\right),
$$

where $D_{+}$and $D_{-}$are the Dirac measures concentrated respectively on +1 and -1 . 
Theorem 3. Let $\mathscr{A}_{\alpha}$ be the Ising automaton. To every input $\varepsilon$ corresponds an output $\delta=\mathscr{A}_{\alpha} \varepsilon$. For almost all $\varepsilon \in\{-1,+1\}^{\mathbb{N}}$,

$$
\lim _{N \rightarrow \infty} \frac{1}{N} \sum_{n=0}^{N-1} \delta_{n}=\alpha .
$$

More generally, for all finite set $Q$ of integers and for almost all $\varepsilon$,

$$
\lim _{N \rightarrow \infty} \frac{1}{N} \sum_{n=0}^{N-1} \prod_{q \in Q}\left(\delta_{n+q}-\alpha\right)=0 .
$$

Proof. Define

$$
F(x)=\operatorname{sgn}(x) \min \{2,|x|\}, \quad x \in \mathbb{R} .
$$

Then

$$
T_{N}(\varepsilon)=\frac{1}{N} \sum_{n=0}^{N-1} \prod_{q \in Q}\left(\delta_{n+1+q}-\alpha\right)=\frac{1}{N} \sum_{n=0}^{N-1} \prod_{q \in Q} \varepsilon_{n+q} F\left(\delta_{n+q}\right) .
$$

Therefore

$$
I(N)=\int\left|T_{N}(\varepsilon)\right|^{2} d \varepsilon=0\left(\frac{1}{N}\right)+\frac{2}{N^{2}} \sum_{m<n} \int \prod_{q \in Q} \varepsilon_{m+q} \varepsilon_{n+q} F\left(\delta_{m+q}\right) F\left(\delta_{n+q}\right) d \varepsilon .
$$

Let $r$ be the largest integer in $Q$. The term $\varepsilon_{n+r} d \varepsilon_{n+r}$ occurs under the integral sign and the index $n+r$ dominates all other indices of the $\varepsilon_{i}$. Hence

$$
\int \varepsilon_{n+r} d \varepsilon_{n+r}=\frac{1-1}{2}=0
$$

factors out so that

$$
I(N)=0\left(\frac{1}{N}\right)
$$

The series

$$
\sum_{N=1}^{\infty} \frac{1}{N} I(N)
$$

converges and a theorem of Beppo Levi ([5], p. 36) then implies that $T_{N}(\varepsilon)$ converges to 0 for almost all $\varepsilon$. Q.E.D.

Remark. Theorems 2 and 3 show that the spatial average

$$
\frac{1}{\left|S_{\alpha}\right|} \sum_{M \in S_{\alpha}} \varphi(M)
$$

and the dynamical average

$$
\lim _{N \rightarrow \infty} \frac{1}{N} \sum_{n=0}^{N-1} \delta_{n}
$$

both coincide. This is of course just an instance of an ergodic principle. 


\section{II.3. Opacity of as Ising Automaton}

A simple adaptation of Theorem 1 shows the following result.

Theorem 4. Let $\mathscr{A}_{\alpha}$ be the Ising automaton. Then

(i) $\omega\left(\mathscr{A}_{0}\right)=1$,

(ii) if $\alpha>0$,

$$
\omega\left(\mathscr{A}_{\alpha}\right)=\sqrt{\frac{\mu-1}{\mu}},
$$

where $\mu=\max \{1,[4 / \alpha]\}$.

Remark 1. As $\alpha$ decreases to $0, \omega\left(\mathscr{A}_{\alpha}\right)$ tends to $1=\omega\left(\mathscr{A}_{0}\right)$. Even though the automaton changes drastically in the neighborhood of $\alpha=0$, the $\operatorname{map} \alpha \mapsto \omega\left(\mathscr{A}_{\alpha}\right)$ is continuous at $\alpha=0$.

Remark 2. Suppose we are given a one-sided Ising chain in which we do not know the distribution $\varepsilon$ of the impurities. Apply a strong external field $H \geqq 2 J$, and read off the equilibrium configuration $\sigma$ : this gives $\varepsilon$.

A variation of the above idea could be as follows. Suppose we wish to measure the binding energy $J$. We start out with a very large external field $H$ and observe the equilibrium spin configuration $\sigma$. As $H$ decreases, at some point $H_{c}, \sigma$ will change. Then $H_{c}=2 J$.

Apology. The one-sided Ising chain may not correspond to a real system. Yet, the ideas developped in these pages originate from physics and the mathematics involved, even though quite simple, appear to us as being interesting in themselves. I mention two related forthcoming articles, the first one by F. Blanchard, J.-M. Dumont, and A. Thomas: Generic sequences, Transducers and Multiplication of Normal Numbers (preprint). The second one is written by A. Broglio and P. Liardet: Predictability (preprint).

Jean-Paul Allouche and Nathalie Loraud have been a great help in preparing this article. May they accept my warmest thanks.

\section{References}

1. Allouche, J.-P., Mendes France, M.: Quasicrystal Ising chain and automata theory. J. Stat. Phys. 42, 809-821 (1986)

2. Bass, J.: Suites uniformément denses, moyennes trigonométriques, fonctions pseudoaléatoires. Bull. Soc. Math. France 87, 1-69 (1959)

3. Derrida, B., Gardner, E.: Metastable states of a spin glass chain at 0 temperature. J. Phys. 47, 959 (1986)

4. Mendes France, M.: Ising transducer. Ann. Inst. Henri Poincaré, Physique Théor. 52, 259-265 (1990)

5. Riesz, F., Nagy, B.Sz.: Leçons d'analyse fonctionnelle, 6e édition. Paris: Gauthier-Villars 1972 